OPEN ACCESS

Edited by:

Jonathan Richard Bishop,

Starship Children's Health,

New Zealand

Reviewed by:

Tudor Lucian Pop,

Iuliu Haţieganu University of Medicine

and Pharmacy, Romania

Batia Weiss,

Edmond and Lily Safra Children's

Hospital, Israel

*Correspondence:

Ming-Wei Lai

a22141@cgmh.org.tw

Tsung-Chieh Yao

yao@cgmh.org.tw

†These authors have contributed equally to this work

Specialty section:

This article was submitted to

Pediatric Gastroenterology,

Hepatology and Nutrition,

a section of the journal

Frontiers in Pediatrics

Received: 10 June 2019 Accepted: 02 October 2019 Published: 30 October 2019

Citation:

Hua M-C, Huang J-L, Hu C-C, Yao T-C and Lai M-W (2019) Including

Fibroblast Growth Factor-21 in Combined Biomarker Panels Improves Predictions of Liver Steatosis Severity

in Children. Front. Pediatr. 7:420.

doi: 10.3389/fped.2019.00420

\section{Including Fibroblast Growth Factor-21 in Combined Biomarker Panels Improves Predictions of Liver Steatosis Severity in Children}

\author{
Man-Chin Hua ${ }^{1,2}$, Jing-Long Huang ${ }^{2,3}$, Ching-Chih $\mathrm{Hu}^{2,4}$, Tsung-Chieh $\mathrm{Yao}^{2,3 *+}$ and \\ Ming-Wei Lai ${ }^{2,5,6 * t}$
}

\begin{abstract}
${ }^{1}$ Department of Pediatrics, Chang Gung Memorial Hospital, Keelung, Taiwan, ${ }^{2}$ Chang Gung University College of Medicine, Taoyuan, Taiwan, ${ }^{3}$ Division of Allergy, Asthma and Rheumatology, Department of Pediatrics, Chang Gung Memorial Hospital, Taoyuan, Taiwan, ${ }^{4}$ Division of Hepatology, Department of Hepatogastroenterology, Chang Gung Memorial Hospital, Keelung, Taiwan, ${ }^{5}$ Division of Gastroenterology, Department of Pediatrics, Chang Gung Memorial Hospital, Taoyuan, Taiwan, ${ }^{6}$ Liver Research Center, Chang Gung Memorial Hospital, Taoyuan, Taiwan
\end{abstract}

Background: Previous studies reported conflicting results regarding the association between fibroblast growth factor-21 (FGF-21) and non-alcoholic fatty liver disease (NAFLD). This study aimed to evaluate the feasibility of combining FGF-21, obesity indices, and biochemical tests for predicting high-grade liver steatosis in children.

Methods: A total of 203 children and adolescents aged 5-18 years were enrolled, and their anthropometric data, body composition, liver ultrasound score for NAFLD (range, 0-6), biochemical test results, and FGF-21, leptin, and adiponectin levels were analyzed. Children were categorized according to body mass index (BMI) and NAFLD scores. Univariate analysis and multivariate linear regression were used to identify independent predictors for the degree of liver steatosis. The accuracy of the models was also evaluated using a receiver-operating characteristic (ROC) curve.

Results: FGF-21 levels were significantly higher in subjects with high-grade liver steatosis $(P<0.001)$. In obese and overweight children, regression analysis indicated that higher BMl and higher gamma-glutamyl transferase $(\gamma-\mathrm{GT})$, triglycerides (TG), and FGF-21 levels were independent risk factors strongly correlated with NAFLD scores. FGF-21 combined with any of the above parameters showed a larger area under the ROC (AUROC, 0.861-0.873) than either parameter used alone. Overall, the best performance was obtained by combing FGF-21, $\gamma$-GT, and TG, with an AUROC of 0.871 , specificity of $82.54 \%$, and sensitivity of $83.78 \%$ for predicting high-grade liver steatosis.

Conclusion: BMI, FGF-21, $\gamma$-GT, and TG levels were strongly correlated with liver steatosis severity. Including FGF-21 in the biomarker panels may improve the accuracy for identifying obese and overweight children with high-grade liver steatosis.

Keywords: FGF-21, biochemical parameters, BMI, combined analysis, predictors, high-grade liver steatosis, liver ultrasound, childhood obesity 


\section{INTRODUCTION}

Concomitant with the rising prevalence of childhood obesity, non-alcoholic fatty liver disease (NAFLD) has been increasingly recognized in children over recent decades (1). NAFLD may progress from steatosis to non-alcoholic steatohepatitis (NASH), fibrosis, and cirrhosis, which may result in liver failure or hepatocellular carcinoma in the long run $(2,3)$. Hence, we must address the silent health threats and need for monitoring of the target group. Although liver histology is the gold standard for differentiating NAFLD from the normal healthy group, it is invasive and impractical for screening purposes. Some studies have surveyed non-invasive parameters including anthropometric measurements (e.g., body mass index [BMI] and waist circumference) (4-6), body fat distribution (e.g., visceral and subcutaneous fat) $(7,8)$, biochemical data (e.g., homeostasis model of assessment values for insulin resistance [HOMA-IR] and hypertriglyceridemia), and adipokine patterns (e.g., leptin and adiponectin) in NAFLD patients (9-12). However, the ideal parameters to differentiate NAFLD from the obese but healthy group or simple steatosis from NASH are still lacking.

Fibroblast growth factor-21 (FGF-21), which is mainly secreted by the liver, adipose tissue, skeletal muscle, and pancreas, has recently been identified to affect glucose and lipid metabolism in hepatocytes and adipocytes $(13,14)$. Some studies reported that FGF-21 protects against hepatic steatosis and damage (14), whereas others presented conflicting results (15-17); thus, the role of FGF-21 on NAFLD requires further investigation.

The present study involved a thorough evaluation of anthropometric data and body composition using different techniques and an examination of the correlation between biochemical markers, such as adipokines and FGF-21 levels, and liver steatosis severity. Here we aimed to explore which parameters were independent predictors for liver steatosis and evaluate the feasibility of combining indices to predict high-grade liver steatosis in overweight and obese children.

\section{METHODS}

\section{Study Subjects}

From March 2015 to August 2017, we recruited obese and overweight children aged 5-18 years who were willing to undergo anthropometric and body composition measurements, blood sampling, and fatty liver sonographic screening at the outpatient clinic of Chang Gung Memorial Hospital, Keelung, and healthy controls from the Prediction of Allergies in Taiwanese Children (PATCH) cohort study (18). Obese children and adolescents with abnormal aminotransferase levels referred to our pediatric gastroenterology outpatient clinic were also recruited. All of the parents and children received verbal and written information about the study's design and objectives. All participants were enrolled after written informed consent was obtained from their parents. Those subjects who received medications that could affect glucose metabolism and those who presented chronic liver diseases, including hepatitis $B$, hepatitis $C$, autoimmune hepatitis, and Wilson's disease, were excluded. This study was approved by the Research Ethics Committee of Chang Gung Memorial
Hospital (104-7100C, 106-3610C, and 104-7145B) and complied with the Declaration of Helsinki.

\section{Anthropometric and Body Composition Measurements}

Body weight (BW) and body height (BH) were measured using an electronic scale (Super-view-HW3050; Hualien, Taiwan; precisions of $0.1 \mathrm{~kg}$ and $0.1 \mathrm{~cm}$, respectively) with the participants wearing light clothes and no shoes. $\mathrm{BH}$ and $\mathrm{BW}$ were used to calculate BMI in $\mathrm{kg} / \mathrm{m}^{2}$. Children with a BMI $\geq 95$ th percentile were defined as obese, those with a BMI $\geq 85-95$ th percentile were defined as overweight, and those with a BMI $=10-85$ th percentile were defined as normal. The age- and sex-specific standards were based on the BMI charts officially declared by Department of Health in Taiwan (19). Waist circumference was measured at the level of the umbilicus. Hip circumference was measured at the point of maximal protrusion of the buttocks. The waist-to-hip and waist-to-height ratios were calculated from these measurements. Two skinfold sites on the bilateral triceps and gastrocnemius were measured with a skinfold caliper (Slim guide, Michigan, USA) to the nearest $0.5 \mathrm{~mm}$ using standard procedures. Mean skinfold thickness values were calculated from skinfold measurements of the bilateral triceps and gastrocnemius.

Body composition was measured using a multi-frequency (20 and $100 \mathrm{kHz}$ ) bioelectrical impedance analysis (BIA) device that uses an 8-point tactile electrode system (InBody 230, Seoul, Korea) to yield detailed data on body composition, including body fat percentage, trunk fat percentage, total body fat, fatfree mass, total body water, and total body muscle (20). The InBody 230 is suitable for individuals aged 3-99 years. All anthropometric measurements and BIA were performed by two well-trained research assistants.

\section{Liver Ultrasonographic Examination and Scoring System for Liver Steatosis}

Liver steatosis severity was determined in each participant using ultrasonography by the same pediatric gastroenterologist (21). Liver steatosis severity was assessed on ultrasonography using three scoring items that have been described by Hamaguchi et al. (22). This score is referred to as the NAFLD score in this study and is composed of: (1) Bright liver and hepatorenal echo contrast (score 0-3). Score 0: both liver echogenicity and hepatorenal contrast was normal; Score 1: either liver echogenicity or hepatorenal contrast was increased; Score 2: both liver echogenicity and hepatorenal contrast were mildly increased; Score 3: both liver echogenicity and hepatorenal contrast were significantly increased; (2) Deep attenuation of the diaphragm (score 0-2). Score 0: the diaphragm could be clearly distinguished by an observer; Score 1: visualization of the diaphragm was obscure, but an observer could distinguish the diaphragm; Score 2: an observer could not distinguish the diaphragm (3) Visualization of the intrahepatic vessels (score, 01). Score 0: no evidence of vessel blurring; Score 1: the border of intrahepatic vessels were unclear and the lumen of intrahepatic vessels were narrowed. Standardized views of the liver were 
obtained to enable scoring of these three items (NAFLD score 06) (22). If the score for the hepatorenal echo contrast and bright liver was $\geq 1$, we summed up all scores and defined the subjects as having liver steatosis. If hepatorenal echo contrast and bright liver scores were zero, the total score was defined as zero (22).

\section{Collection of Biochemical Data}

Blood samples (10-mL aliquots) were collected in test tubes containing ethylenediaminetetraacetic acid (1g/L) after the subjects fasted overnight, and the following markers were analyzed: alanine aminotransferase (ALT), aspartate aminotransferase (AST), gamma-glutamyl transferase ( $\gamma$-GT), glucose, insulin, triglyceride (TG), total cholesterol, low-density lipoprotein cholesterol (LDL-C), and high-density lipoprotein cholesterol (HDL-C). HOMA-IR was also obtained (23). The diagnoses of hepatitis $\mathrm{B}$ and $\mathrm{C}$, autoimmune hepatitis, and Wilson's disease were excluded using the appropriate diagnostic tests.

\section{Measurement of FGF-21, Adiponectin, and Leptin}

Plasma was separated by centrifugation (3,000 rpm for $10 \mathrm{~min}$ ) at room temperature within $3 \mathrm{~h}$ of blood collection and stored at $-80^{\circ} \mathrm{C}$ until use. After the samples were thawed, the concentrations of FGF-21 (pg/mL), adiponectin $(\mathrm{ng} / \mathrm{mL})$, and leptin $(\mathrm{pg} / \mathrm{mL})$ were determined using enzyme-linked immunosorbent assay kits (R\&D Systems, Minneapolis, MN, USA) according to the manufacturer's instructions. The assays had a minimum detection limit of $8.69 \mathrm{pg} / \mathrm{mL}$ for FGF-21, $0.891 \mathrm{ng} / \mathrm{mL}$ for adiponectin, and $7.8 \mathrm{pg} / \mathrm{mL}$ for leptin.

\section{Group Definitions}

\section{Obese Children With Liver Steatosis}

Obese (BMI $\geq 95$ th percentile) or overweight (BMI $\geq 85-95$ th percentile) children with an NAFLD score $\geq 1$. Specifically, an NAFLD score of 4-6 was defined as high-grade liver steatosis, while an NAFLD score of 1-3 was defined as low-grade liver steatosis.

\section{Simple Obese Children}

Obese (BMI $\geq 95$ th percentile) or overweight (BMI $\geq 85-95$ th percentile) children with an NAFLD score equal to 0 .

\section{Healthy Controls}

Healthy children with a normal BMI (BMI $=10-84$ th percentile), NAFLD score $=0$, and no medical disorders.

\section{Statistical Analysis}

Continuous and normally distributed variables are expressed as mean $\pm \mathrm{SD}$ and were analyzed using one-way analysis of variance. For continuous variables with a non-normal distribution, the differences between two study groups were estimated using the Mann-Whitney $U$ test. In obese and overweight children, the relationship between NAFLD score and predictive parameters, such as obesity indices (e.g., BMI, waistto-hip ratio, waist-to-height ratios, mean skinfold thickness, and body fat composition), biochemical data, and the logarithmically transformed concentrations of FGF-21, adiponectin, and leptin were determined using a univariate linear regression model. The parameters with values of $P<0.001$ on univariate logistic regression analysis were entered in the stepwise linear regression model. Finally, the accuracy of the models was evaluated using the area under a receiver operating characteristic (AUROC) curve with 95\% confidence interval (95\% CI) and the sensitivity and specificity of the final model were calculated. For combined analysis with normally and nonnormally distributed variables, all values in the ROC models were logarithmically transformed in the statistics. Two-tailed $P$ values $<0.05$ were considered significant. The statistical analysis was performed using SPSS Statistics version 20 (IBM, Armonk, NY, USA).

\section{RESULTS}

\section{Subject Characteristics}

A total of 203 children and adolescents (102 male, 101 female; mean age, $13.69 \pm 3.16$ years) were enrolled and divided into healthy controls $(n=89)$, simple obese $(n=31)$, and obese with liver steatosis $(n=83)$ groups. There were no intergroup differences in mean age or sex. Eighty-three of the $114(72.8 \%)$ obese or overweight children presented with liver steatosis, of whom $42(50.6 \%)$ were defined as having lowgrade steatosis and $41(49.4 \%)$ were defined as having high-grade liver steatosis.

\section{Intergroup Comparison of Obesity Indices and Biochemical Data}

As shown in Table 1, a significant and progressive increase in BMI, waist circumference, waist-to-hip ratio, and waistto-height ratio was found from the healthy control to the simple obese to the obese with steatosis groups $(P<$ 0.001). With regard to body composition data measured by BIA, body fat percentage, trunk fat percentage, and total body fat weight were significantly different across the three groups $(P<0.001)$. Moreover, the HOMA-IR index, AST, ALT, $\gamma$-GT, and TG levels were significantly higher, whereas the HDL-C levels were significantly lower in obese children with liver steatosis compared with the healthy controls and simple obese children $(P<0.001)$ (Table 1).

\section{Intergroup Comparison of FGF-21, Adiponectin, and Leptin Levels}

On the Mann-Whitney U-test, FGF-21 levels were significantly higher in the high-grade liver steatosis group than in the lowgrade liver steatosis group, simple obese children, and healthy controls (median [IQR], 183.17 [113.03-301.14] pg/mL vs. 86.58 [62.76-183.02] pg/mL vs. 79.76 [30.91-146.76] pg/mL vs. 53.45 [29.78-87.21] pg/mL, $P<0.001$, respectively) (Figure 1A). Adiponectin levels tended to decrease as the hepatic fat content was increased. Adiponectin levels were significantly lower in the low- and high-grade liver steatosis groups than in the healthy controls (median [IQR], 2.86 [1.93-5.92] $\mu \mathrm{g} / \mathrm{mL}$ vs. 6.15 [2.75-9.22] $\mu \mathrm{g} / \mathrm{mL}, P=0.036$; and 2.81 [1.98-3.72] $\mu \mathrm{g} / \mathrm{mL}$ vs. $6.15[2.75-9.22] \mu \mathrm{g} / \mathrm{mL}, P<0.001$, respectively) (Figure 1B). 
TABLE 1 | Comparison of clinical and laboratory parameters in healthy controls, simple obese children, and obese children with liver steatosis.

\begin{tabular}{|c|c|c|c|}
\hline & $\begin{array}{l}\text { Healthy } \\
\text { controls } \\
(N=89)\end{array}$ & $\begin{array}{l}\text { Simple obese } \\
\text { children } \\
(N=31)\end{array}$ & $\begin{array}{l}\text { Obese children } \\
\text { with liver } \\
\text { steatosis } \\
(N=83)\end{array}$ \\
\hline \multicolumn{4}{|c|}{ BASIC AND ANTHROPOMETRIC DATA } \\
\hline Age (y) & $14.06 \pm 3.97$ & $13.74 \pm 3.60$ & $12.78 \pm 3.27$ \\
\hline Gender, male (\%) & $43(48.3)$ & $13(41.9)$ & $46(55.4)$ \\
\hline BMl $\left(\mathrm{kg} / \mathrm{m}^{2}\right)$ & $18.69 \pm 2.21$ & $24.54 \pm 3.54^{\mathrm{a}}$ & $27.30 \pm 3.53^{\mathrm{ab}}$ \\
\hline Waist circumference (cm) & $66.72 \pm 8.43$ & $80.76 \pm 11.91^{\mathrm{a}}$ & $88.40 \pm 14.83^{\mathrm{ab}}$ \\
\hline Hip circumference (cm) & $85.10 \pm 11.32$ & $97.03 \pm 12.65^{a}$ & $99.00 \pm 14.08^{a}$ \\
\hline Waist-hip ratio & $0.79 \pm 0.06$ & $0.83 \pm 0.07^{a}$ & $0.90 \pm 0.12^{\mathrm{ab}}$ \\
\hline Waist-height ratio & $0.43 \pm 0.04$ & $0.51 \pm 0.05^{a}$ & $0.57 \pm 0.09^{a b}$ \\
\hline $\begin{array}{l}\text { Mean skinfold thickness } \\
(\mathrm{cm})\end{array}$ & $1.52 \pm 1.53$ & $1.82 \pm 0.70$ & $2.02 \pm 1.32^{\mathrm{a}}$ \\
\hline \multicolumn{4}{|l|}{ DATA FROM BIA } \\
\hline Body fat (\%) & $20.83 \pm 7.50$ & $31.56 \pm 8.06^{\mathrm{a}}$ & $37.27 \pm 7.31^{\mathrm{ab}}$ \\
\hline Trunk fat (\%) & $18.63 \pm 9.57$ & $32.20 \pm 8.58^{a}$ & $38.09 \pm 7.10^{\mathrm{ab}}$ \\
\hline Total body fat $(\mathrm{kg})$ & $10.17 \pm 5.98$ & $20.03 \pm 7.40^{\mathrm{a}}$ & $25.12 \pm 7.35^{\mathrm{ab}}$ \\
\hline Fat-free mass (kg) & $36.34 \pm 11.58$ & $42.51 \pm 13.20^{a}$ & $41.97 \pm 11.57^{a}$ \\
\hline Total body water (kg) & $26.65 \pm 8.51$ & $31.15 \pm 9.64^{a}$ & $30.73 \pm 8.42^{\mathrm{a}}$ \\
\hline Total body muscle (kg) & $19.56 \pm 6.98$ & $23.25 \pm 7.95^{\mathrm{a}}$ & $23.00 \pm 7.02^{\mathrm{a}}$ \\
\hline \multicolumn{4}{|l|}{ BIOCHEMISTRY DATA } \\
\hline $\begin{array}{l}\text { Fasting blood glucose } \\
(\mathrm{mg} / \mathrm{dL})\end{array}$ & $86.21 \pm 17.37$ & $89.36 \pm 5.08$ & $90.69 \pm 7.37$ \\
\hline Insulin ( $\mu|U / m|)$ & $4.92 \pm 3.62$ & $8.35 \pm 5.72$ & $14.87 \pm 10.27^{a}$ \\
\hline HOMA-IR & $1.39 \pm 0.69$ & $2.57 \pm 1.26$ & $3.69 \pm 2.88^{\mathrm{ab}}$ \\
\hline $\mathrm{ALT}(\mathrm{U} / \mathrm{L})$ & $14.07 \pm 6.86$ & $17.41 \pm 11.03$ & $50.18 \pm 15.94^{a b}$ \\
\hline AST (U/L) & $18.69 \pm 6.82$ & $18.04 \pm 4.78$ & $33.77 \pm 23.32^{\mathrm{ab}}$ \\
\hline$\gamma-\mathrm{GT}(\mathrm{U} / \mathrm{L})$ & $13.72 \pm 5.68$ & $15.17 \pm 4.72$ & $24.78 \pm 12.63^{\mathrm{ab}}$ \\
\hline Triglycerides (mg/dL) & $72.01 \pm 62.47$ & $80.52 \pm 40.29$ & $117.76 \pm 60.2^{\mathrm{ab}}$ \\
\hline Total cholesterol (mg/dL) & $167.97 \pm 34.33$ & $162.78 \pm 32.55$ & $176.21 \pm 26.10$ \\
\hline HDL-C (mg/dL) & $55.28 \pm 15.71$ & $55.32 \pm 14.04$ & $44.75 \pm 8.00^{\mathrm{ab}}$ \\
\hline LDL-C (mg/dL) & $94.27 \pm 25.80$ & $101.92 \pm 27.78$ & $106.65 \pm 23.73$ \\
\hline
\end{tabular}

$P$-value based on ANOVA test with multiple comparison analysis.

a'ndicated significant differences ( $P$-values $<0.05$ ) between liver steatosis group (or simple obese group) and healthy control group.

${ }^{b}$ Indicated significant differences ( $P$-values $\left.<0.05\right)$ between liver steatosis group and simple obese group.

In contrast, the leptin levels in the study subjects were variable. Compared to those in the healthy controls, the leptin levels were significantly higher in the low-grade liver steatosis group (median [IQR], 20.06 [10.26-22.61] ng/mL vs. 6.84 [1.48-10.26] ng/mL, $P$ $<0.001$ ) but not in the high-grade liver steatosis group (median [IQR], 10.97 [5.13-20.51] ng/mL vs. 6.84 [1.48-10.26] ng/mL, $P$ $=0.435)$ (Figure 1C).

\section{Identification of Independent Predictors for Liver Steatosis Grading in Obese and Overweight Children}

Variables associated with liver steatosis grade (NAFLD scores) were first assessed by univariate analysis. Considering that the levels of FGF-21, adiponectin, and leptin were not normally distributed, values were logarithmically transformed to continuous variables in the statistical models. As shown in Table 2, BMI and body fat mass, including body fat percentage, trunk fat percentage, and total body fat weight, showed a strong correlation with liver steatosis grade $(B$ $=0.400-0.493, P<0.001)$, whereas the waist-to-hip and waist-to-height ratios showed a moderate correlation with steatosis grade $(B=0.252-0.315, P<0.01)$ (Table 2). All tested biochemical parameters were significantly associated with steatosis grade. Of these, AST, ALT, $\gamma$-GT, TG, and total cholesterol showed a strong positive correlation $(B=0.354-$ 0.603, $P<0.001)$, HOMA-IR and LDL-C showed a moderate positive correlation $(B=0.332-0.395, P<0.01)$, and HDL$C$ levels showed an inverse correlation with liver steatosis degree $(B=-0.308, P=0.003$ ) (Table 2). Moreover, the logarithmically transformed FGF-21 levels were positively and significantly correlated with liver steatosis grade $(B=0.516, P$ $<0.001)$ whereas logarithmically transformed adiponectin $(B=$ $-0.217, P=0.067)$ and leptin levels $(B=0.003, P=0.978)$ were not.

We next used multivariate regression analysis with a stepwise procedure to assess the best predictors for differentiating liver steatosis severity in obese and overweight children (Table 2). The results indicated that stepwise increments in BMI $(B$ $=0.174, P=0.033), \gamma$-GT $(B=0.368, P<0.001)$, TG $(B=0.180, P=0.035)$, and log-transformed FGF-21 levels $(B=0.273, P=0.002)$ were significantly correlated the steatosis grade of obese and overweight children $(P<0.05)$ (Table 2).

\section{Evaluation of Accuracy of Single or Combined Tests to Predict High-Grade Liver Steatosis}

As shown in Table 3 and Figure 2, the ROC curve analysis of high-grade liver steatosis in obese and overweight children revealed that $\gamma$-GT $\geq 21.50 \mathrm{U} / \mathrm{L}, \mathrm{FGF}-21 \geq 106.10 \mathrm{pg} / \mathrm{mL}, \mathrm{TG} \geq$ $77.00 \mathrm{mg} / \mathrm{dL}$, and $\mathrm{BMI} \geq 25.83 \mathrm{~kg} / \mathrm{m}^{2}$ were significant predictors with optimal AUROC (0.732-0.840) and good sensitivity (82.5$90.24 \%$ ); nevertheless, the specificity was relatively lower (50.0$70.50 \%)$. Of these four indices, $\gamma$-GT was the best predictor with an AUROC of 0.840 (95\% CI, 0.765-0.915), sensitivity of $82.50 \%$, and specificity of $70.50 \%$. Our further analysis found that the combination of each biomarker with FGF21 demonstrated better accuracy with an AUROC of 0.8610.873 and higher specificity of $72.88-82.54 \%$ than a single test. Overall, the best performance was obtained by combing FGF$21, \gamma-G T$, and TG with the AUROC of 0.871 , specificity of $82.54 \%$, and sensitivity of $83.78 \%$ for predicting high-grade liver steatosis (Figure 2B).

\section{DISCUSSION}

In this study, we demonstrated that $\gamma$-GT, FGF-21, TG, and BMI were significantly associated with liver steatosis severity 


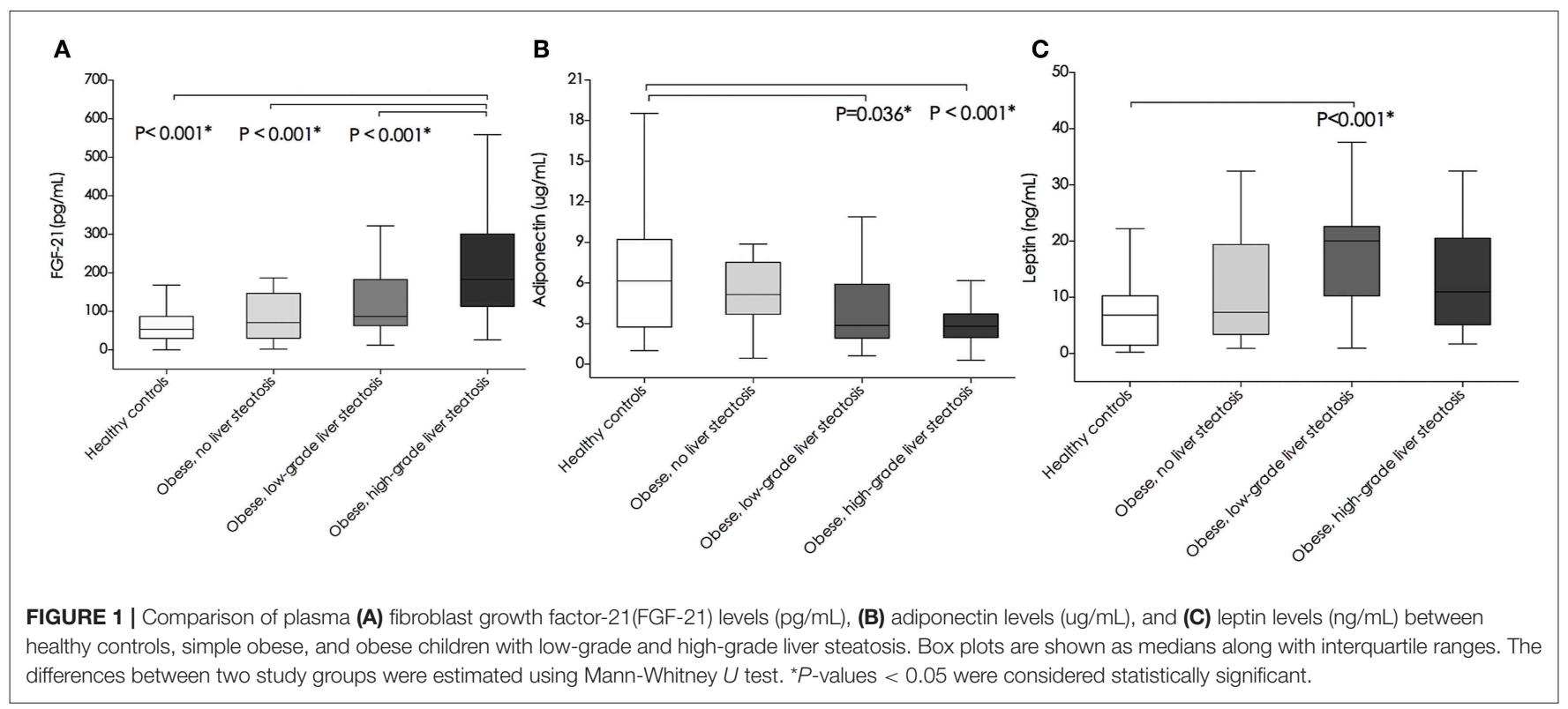

TABLE 2 | Regression analysis of clinical and laboratory parameters in relation to NAFLD score in obese and overweight children.

\begin{tabular}{|c|c|c|c|c|c|c|}
\hline & \multicolumn{3}{|c|}{ Univariate regression analysis } & \multicolumn{3}{|c|}{ Multiple linear regression with a stepwise procedure } \\
\hline BMI $\left(\mathrm{kg} / \mathrm{m}^{2}\right)$ & 0.493 & $0.182-0.362$ & $<0.001$ & 0.174 & $0.009-0.207$ & 0.033 \\
\hline Waist-hip ratio & 0.252 & $1.206-7.863$ & 0.008 & & & \\
\hline Waist-height ratio & 0.315 & $3.160-11.708$ & 0.001 & & & \\
\hline Total body fat (\%) & 0.429 & $0.067-0.155$ & $<0.001$ & & & \\
\hline Trunk fat (\%) & 0.443 & $0.071-0.158$ & $<0.001$ & & & \\
\hline Total body fat (kg) & 0.400 & $0.061-0.153$ & $<0.001$ & & & \\
\hline Fat-free mass (kg) & 0.006 & $-0.031-0.033$ & 0.952 & & & \\
\hline Total body water (kg) & 0.006 & $-0.043-0.046$ & 0.949 & & & \\
\hline $\operatorname{ALT}(\mathrm{U} / \mathrm{L})$ & 0.580 & $0.018-0.032$ & $<0.001$ & & & \\
\hline$\gamma-\mathrm{GT}(\mathrm{U} / \mathrm{L})$ & 0.603 & $0.074-0.128$ & $<0.001$ & 0.368 & $0.031-0.088$ & $<0.001$ \\
\hline Triglyceride (mg/dL) & 0.452 & $0.010-0.022$ & $<0.001$ & 0.180 & $0.000-0.013$ & 0.035 \\
\hline Total cholesterol (mg/dL) & 0.354 & $0.013-0.038$ & $<0.001$ & & & \\
\hline $\mathrm{HDL}-\mathrm{C}(\mathrm{mg} / \mathrm{dL})$ & -0.308 & -0.100 to -0.022 & 0.003 & & & \\
\hline LDL-C (mg/dL) & 0.332 & $0.010-0.042$ & 0.001 & & & \\
\hline \multicolumn{7}{|l|}{ POTENTIAL BIOMARKERS } \\
\hline Log FGF-21 (pg/mL) & 0.516 & $1.622-3.213$ & $<0.001$ & 0.273 & 0.510-2.099 & 0.002 \\
\hline Log adiponectin (ug/mL) & -0.217 & $-2.576-0.101$ & 0.067 & & & \\
\hline Log leptin (ng/mL) & 0.003 & $-1.060-1.089$ & 0.978 & & & \\
\hline
\end{tabular}

P-value based on logistic regression analysis; P-values < 0.05 were considered statistically significant; Cl, Confidence interval.

in obese and overweight children and may be used as the initial assessment. In addition, the combination of FGF21 with these biochemical parameters can further improve the accuracy for predicting high-grade liver steatosis than either test used alone. Overall, the combination of FGF$21, \gamma-G T$, and TG showed the best performance. Taken together, our results support the potential role of FGF-21 in pediatric NAFLD. 
TABLE 3 | Summary receiver's operating characteristic (ROC) of variables in the prediction of high-grade liver steatosis in the obese and overweight subjects.

\begin{tabular}{|c|c|c|c|c|c|c|}
\hline Single blood test & AUROC & Cut-off value & $95 \% \mathrm{Cl}$ & Sensitivity\% & Specificity\% & $P$-value \\
\hline$\gamma$-GT (U/L) & 0.840 & 21.50 & $0.765-0.915$ & 82.50 & 70.50 & $<0.001$ \\
\hline FGF-21 (pg/mL) & 0.781 & 106.10 & $0.687-0.874$ & 86.49 & 60.00 & $<0.001$ \\
\hline $\mathrm{BMI}\left(\mathrm{kg} / \mathrm{m}^{2}\right)$ & 0.748 & 25.83 & $0.657-0.839$ & 82.93 & 55.56 & $<0.001$ \\
\hline $\mathrm{TG}(\mathrm{mg} / \mathrm{dL})$ & 0.732 & 77.00 & $0.639-0.824$ & 90.24 & 50.00 & $<0.001$ \\
\hline Combined tests & AUROC & Cut-off value (Log) & $95 \% \mathrm{Cl}$ & Sensitivity\% & Specificity\% & $P$-value \\
\hline FGF-21 and $\gamma$-GT & 0.861 & 3.318 & $0.786-0.937$ & 89.19 & 74.60 & $<0.001$ \\
\hline FGF-21 and $\gamma$-GT and TG & 0.871 & 5.403 & $0.801-0.942$ & 83.78 & 82.54 & $<0.001$ \\
\hline FGF-21 and $\gamma$-GT and TG and BMI & 0.873 & 6.661 & $0.801-0.945$ & 94.59 & 72.88 & $<0.001$ \\
\hline
\end{tabular}

TG, triglyceride; $\mathrm{Cl}$, confidence interval.

$P$-value based on ROC. P-values $<0.05$ were considered statistically significant.

Combined tests: all values were logarithmically transformed in the statistical models.

Recently, relationships between higher FGF-21 levels and adverse lipid profiles, obesity, metabolic syndrome, or type 2 diabetes mellitus have been reported in adults (24). However, the role of FGF-21 in the development of NAFLD is not well-understood. Evidence from studies in adults show that elevated FGF-21 levels reduce hepatic lipogenesis and improve insulin sensitivity $(25,26)$ and are negatively correlated with the probability of NAFLD and NASH (14). Nonetheless, several studies of pediatric patients, including our own, seem to contradict these results $(13,15,17)$. In an ultrasonographybased 3-years prospective study, Li et al. reported that children who progressed to NAFLD had significantly higher baseline and follow-up FGF-21 levels than those who did not, suggesting that baseline FGF-21 level is an independent predictor of NAFLD (27). In another pediatric study, Giannini et al. reported that FGF-21 levels were increased in obese children with high hepatic fat content in MRI and significantly correlated with the NAFLD severity score in liver specimens (28). A possible explanation for the increase in FGF-21 concentration in our subjects with NAFLD may be due to a feedbackinduced protective response against hepatic steatosis or an FGF-21-resistant state $(15,26)$. As measuring FGF-21 levels is simple, quick, and inexpensive, we suggest that FGF-21 can be used as a biomarker for predicting pediatric NAFLD in the future (29).

Our data revealed that no single test had good sensitivity and specificity for predicting liver steatosis severity, whereas combined biomarker panels showed better performance. Among the biochemical parameters, $\gamma$-GT was the best predictor for high-grade liver steatosis in our study. As a high $\gamma$-GT has been shown to be associated with advanced fibrosis in NAFLD (30), the higher $\gamma$-GT in our subjects with highgrade liver steatosis imply the possibility of progressive liver fibrosis (31). Notably, AST and ALT were not identified as independent predictors for liver steatosis grading in the logistic regression model. Previous studies also reported that approximately $80 \%$ of NAFLD subjects had normal ALT levels (32), indicating that ALT is not justified as an independent test for screening for high-grade steatosis in children (33).

Increasing evidence indicates that adipose tissue can produce multiple adipokines that trigger inflammatory processes, insulin resistance, and dyslipidemia $(7,24,34,35)$ as well as contribute to pediatric NAFLD and high-grade liver steatosis $(18,35,36)$. In this study, an increased BMI showed better discriminative ability for high-grade liver steatosis than other obesity indices (e.g., waist circumference, waist-to-hip ratio, and waist-toheight ratio). BMI is easy to measure; nonetheless, the specificity $(55.56 \%)$ is insufficient. Thus, the measurement of BMI in combination with the above laboratory parameters is recommended.

The strength of this study lies in our thorough evaluation of different clinical and laboratory parameters and the inclusion of FGF-21 as part of a biomarker panel to predict liver steatosis severity. To the best of our knowledge, only a few pediatric studies have evaluated the discriminative capability of FGF-21 based on its sensitivity and specificity for $\operatorname{NAFLD}(27,29)$, and none of the pediatric studies have combined FGF-21 with other serum biomarkers for predicting liver steatosis severity (29). Abdominal ultrasonography is broadly used as a screening method for hepatic steatosis (37). In the present study, we applied a scoring system to provide detailed information about liver steatosis severity (NAFLD scores, $0-6$ ), which is different from other ultrasound-based studies involving children $(10,12,15$, 16). In addition, the NAFLD scores in each participant were scored by the same pediatric gastroenterologist in an attempt to reduce the bias. The limitations are mainly linked to the cross-sectional nature of the study, and we did not perform liver biopsy in these cases. Therefore, whether FGF21 levels are correlated with the long-term outcome or biopsy-proven NASH stages was not confirmed. Further studies to explore serial samples of FGF-21 levels and the association with histology of fatty liver disease severity and longitudinal outcome may increase the significance of these findings. Furthermore, the study sample size was relatively small, and ultrasound did have limitations for detecting mild liver steatosis, which might have 

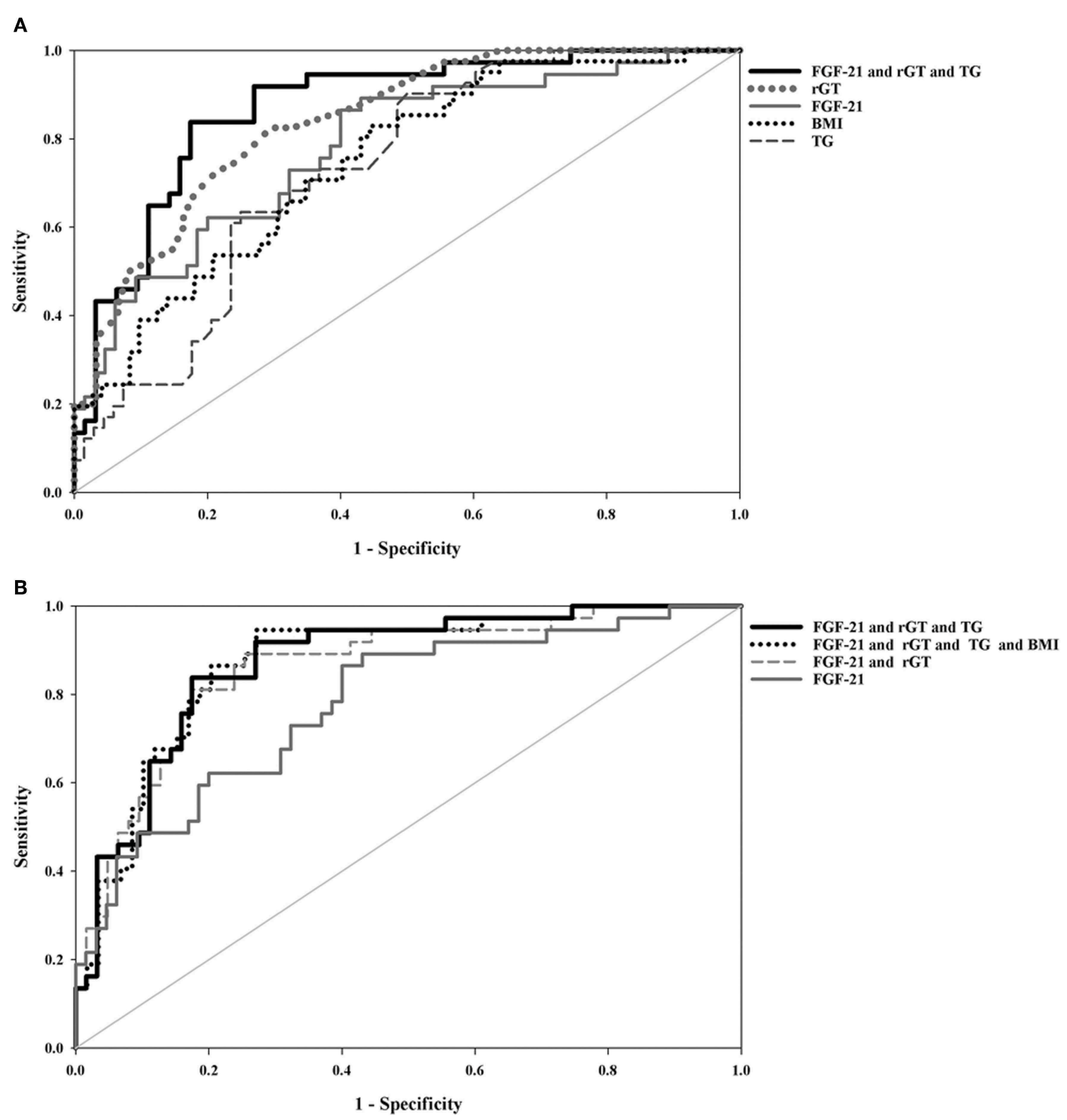

FIGURE 2 | Comparison of area under the receiver-operating characteristic(AUROC) curves between (A) single blood test and a combined biomarker panel (B) combined biomarker panels and FGF-21 for the prediction of high-grade liver steatosis in the obese and overweight subjects. All values were logarithmically transformed in the statistical models.

influenced the results. More cases are required to validate our findings, including the BMI, FGF-21, $\gamma$-GT, and TG cut-off values for detecting high-grade liver steatosis in obese and overweight children and confirming the role of FGF-21 in clinical practice.

\section{CONCLUSION}

Our results demonstrated that $\gamma$-GT, FGF-21, and TG levels and BMI were strongly correlated with liver steatosis severity. Combining FGF-21 and the above biochemical parameters as a biomarker panel may help identify obese and overweight children at risk of developing high-grade liver steatosis.

\section{DATA AVAILABILITY STATEMENT}

The datasets generated for this study are available on request to the corresponding author. 


\section{ETHICS STATEMENT}

This study was approved by the Research Ethics Committee of Chang Gung Memory Hospital (104-7100C, 106-3610C, and 1047145B) and complied with the declaration of Helsinki. Written informed parental consent was obtained.

\section{AUTHOR CONTRIBUTIONS}

$\mathrm{M}-\mathrm{CH}, \mathrm{J}-\mathrm{LH}, \mathrm{C}-\mathrm{CH}, \mathrm{T}-\mathrm{CY}$, and $\mathrm{M}-\mathrm{WL}$ were involved in the study design, participates recruitment, and written consent. $\mathrm{M}-\mathrm{CH}$ involved in the laboratory work, statistical analysis, and interpretation of its results. $\mathrm{M}-\mathrm{CH}$ wrote the first draft of the manuscript. M-WL and T-CY edited the first draft of the

\section{REFERENCES}

1. Welsh JA, Karpen S, Vos MB. Increasing prevalence of nonalcoholic fatty liver disease among United States adolescents, 1988-1994 to 2007-2010. J Pedatr. (2013) 162:496-500. doi: 10.1016/j.jpeds.2012.08.043

2. Yki-Järvinen $\mathrm{H}$. Non-alcoholic fatty liver disease as a cause and a consequence of metabolic syndrome. Lancet Diabetes Endocrinol. (2014) 2:901-10. doi: 10.1016/S2213-8587(14)70032-4

3. Ekstedt M, Franzén LE, Mathiesen UL, Thorelius L, Holmqvist M, Bodemar $\mathrm{G}$, et al. Long-term follow-up of patients with NAFLD and elevated liver enzymes. Hepatology. (2006) 44:865-73. doi: 10.1002/hep.21327

4. Lemoine M, Ratziu V, Kim M, Maachi M, Wendum D, Paye F, et al. Serum adipokine levels predictive of liver injury in nonalcoholic fatty liver disease. Liver Int. (2009) 29:1431-8. doi: 10.1111/j.1478-3231.2009.02022.x

5. Campos GM, Bambha K, Vittinghoff E, Rabl C, Posselt AM, Ciovica R, et al. A clinical scoring system for predicting nonalcoholic steatohepatitis in morbidly obese patients. Hepatology. (2008) 47:1916-23. doi: 10.1002/hep.22241

6. Palekar NA, Naus R, Larson SP, Ward J, Harrison SA. Clinical model for distinguishing nonalcoholic steatohepatitis from simple steatosis in patients with nonalcoholic fatty liver disease. Liver Int. (2006) 26:151-6. doi: 10.1111/j.1478-3231.2005.01209.x

7. Després JP, Lemieux I. Abdominal obesity and metabolic syndrome. Nature. (2006) 444:881-7. doi: 10.1038/nature05488

8. Wajchenberg BL. Subcutaneous and visceral adipose tissue: their relation to the metabolic syndrome. Endocr Rev. (2000) 21:697-738. doi: 10.1210/edrv.21.6.0415

9. Machado MV, Coutinho J, Carepa F, Costa A, Proença H, Cortez-Pinto H. How adiponectin, leptin, and ghrelin orchestrate together and correlate with the severity of nonalcoholic fatty liver disease. Eur J Gastroenterol Hepatol. (2012) 24:1166-72. doi: 10.1097/MEG.0b013e32835609b0

10. Silveira LS, Monteiro PA, Antunes Bde M, Seraphim PM, Fernandes RA, Christofaro DG, et al. Intra-abdominal fat is related to metabolic syndrome and non-alcoholic fat liver disease in obese youth. BMC Pediatr. (2013) 13:115. doi: 10.1186/1471-2431-13-115

11. Nobili V, Manco M, Ciampalini P, Diciommo V, Devito R, Piemonte F, et al. Leptin, free leptin index, insulin resistance and liver fibrosis in children with non-alcoholic fatty liver disease. Eur J Endocrinol. (2006) 155:735-43. doi: $10.1530 /$ eje.1.02288

12. Louthan MV, Barve S, McClain CJ, Joshi-Barve S. Decreased serum adiponectin: an early event in pediatric nonalcoholic fatty liver disease. J Pediatr. (2005) 147:835-8. doi: 10.1016/j.jpeds.2005. 07.030

13. Morris-Stiff G, Feldstein AE. Fibroblast growth factor 21 as a biomarker for NAFLD: integrating pathobiology into clinical practice. J Hepatol. (2010) 53:795-6. doi: 10.1016/j.jhep.2010.07.003

14. Alisi A, Ceccarelli S, Panera N, Prono F, Petrini S, De Stefanis C, et al. Association between serum atypical fibroblast growth factors 21 and 19 manuscript. All authors reviewed the manuscript and approved the final version of the manuscript.

\section{FUNDING}

This study was funded by grants from Chang Gung Memorial Hospital (CMRPG3F0751-52, CMRPG 2E0131-35). The funders had no role in the design of the study and collection, analysis and interpretation of data and in writing the manuscript.

\section{ACKNOWLEDGMENTS}

All authors are grateful to the study participants and their parents, and to Yi-Ling Hsiao and Yi-Wen Lin for data collection and technical assistance.

and pediatric nonalcoholic fatty liver disease. PLoS ONE. (2013) 8:e67160. doi: 10.1371/journal.pone.0067160

15. Li H, Fang Q, Gao F, Fan J, Zhou J, Wang X, et al. Fibroblast growth factor 21 levels are increased in nonalcoholic fatty liver disease patients and are correlated with hepatic triglyceride. J Hepatol. (2010) 53:934-40. doi: 10.1016/j.jhep.2010.05.018

16. Reinehr T, Woelfle J, Wunsch R, Roth CL. Fibroblast growth factor 21 (FGF21) and its relation to obesity, metabolic syndrome, and nonalcoholic fatty liver in children: a longitudinal analysis. J Clin Endocrinol Metab. (2012) 97:2143-50. doi: 10.1210/jc.2012-1221

17. Wu G, Li H, Fang Q, Zhang J, Zhang M, Zhang L, et al. Complementary role of fibroblast growth factor 21 and cytokeratin 18 in monitoring the different stages of nonalcoholic fatty liver disease. Sci Rep. (2017) 7:5095. doi: 10.1038/s41598-017-05257-5

18. Yao TC, Ou LS, Yeh KW, Lee WI, Chen LC, Huang JL. Associations of age, gender, and BMI with prevalence of allergic diseases in children: PATCH study. J Asthma. (2011) 48:503-10. doi: 10.3109/02770903.2011.576743

19. Chen W, Chang MH. New growth charts for Taiwanese children and adolescents based on World Health Organization standards and health related physical fitness. Pediatr Neonatol. (2010) 51:69-79. doi: 10.1016/S1875-9572(10)60014-9

20. Lee LW, Liao YS, Lu HK, Hsiao PL, Chen YY, Chi CC, et al. Validation of two portable bioelectrical impedance analyses for the assessment of body composition in school age children. PLOS ONE. (2017) 12:e0171568. doi: 10.1371/journal.pone.0171568

21. Hua MC, Su HM, Yao TC, Kuo ML, Lai MW, Tsai MH, et al. Alternation of plasma fatty acids composition and desaturase activities in children with liver steatosis. PLoS ONE. (2017) 12:e0182277. doi: 10.1371/journal.pone.0182277

22. Hamaguchi M, Kojima T, Itoh Y, Harano Y, Fujii K, Nakajima T, et al. The severity of ultrasonographic findings in nonalcoholic fatty liver disease reflects the metabolic syndrome and visceral fat accumulation. Am J Gastroentol. (2007) 102:2708-15. doi: 10.1111/j.1572-0241.2007.01526.x

23. Matthews DR, Hosker JP, Rudenski AS, Naylor BA, Treacher DF, Turner RC. Homeostasis model assessment: insulin resistance and Beta-cell function from fasting plasma glucose and insulin concentrations in man. Diabetologia. (1985) 28:412-9. doi: 10.1007/BF00280883

24. Ronti T, Lupattelli G, Mannarino E. The endocrine function of adipose tissue: an update. Clin Endocrinol (Oxf). (2006) 64:355-65. doi: 10.1111/j.1365-2265.2006.02474.x

25. Fisher FM, Chui PC, Nasser IA, Popov Y, Cunniff JC, Lundasen T, et al. Fibroblast growth factor 21 limits lipotoxicity by promoting hepatic fatty acid activation in mice on methionine and choline-deficient diets. Gastroenterology. (2014) 147:1073-83.e6. doi: 10.1053/j.gastro.2014.07.044

26. Xu J, Lloyd DJ, Hale C, Stanislaus S, Chen M, Sivits G, et al. Fibroblast growth factor 21 reverses hepatic steatosis, increases energy expenditure, and improves insulin sensitivity in diet-induced obese mice. Diabetes. (2009) 58:250-9. doi: 10.2337/db08-0392 
27. Li H, Dong K, Fang Q, Hou X, Zhou M, Bao Y, et al. High serum level of fibroblast growth factor 21 is an independent predictor of non-alcoholic fatty liver disease: a 3-year prospective study in China. J Hepatol. (2013) 58:557-63. doi: 10.1016/j.jhep.2012.10.029

28. Giannini C, Feldstein AE, Santoro N, Kim G, Kursawe R, Pierpont B, et al. Circulating levels of FGF-21 in obese youth: associations with liver fat content and markers of liver damage. J Clin Endocrinol Metab. (2013) 98:2993-3000. doi: 10.1210/jc.2013-1250

29. He L, Deng L, Zhang Q, Guo J, Zhou J, Song W, et al. Diagnostic Value of CK-18, FGF-21, and related biomarker panel nonalcoholic fatty liver disease: a systemic review and meta-analysis. Biomed Res Int. (2017) 2017:9729107. doi: 10.1155/2017/9729107

30. Tahan V, Canbakan B, Balci H, Dane F, Akin H, Can G, et al. Serum gammaglutamyltranspeptidase distinguishes non-alcoholic fatty liver disease at high risk. Hepatogastroenterology. (2008) 55:1433-8.

31. Schwimmer JB, Newton KP, Awai HI, Choi LJ, Garcia MA, Ellis LL, et al. Paediatric gastroenterology evaluation of overweight and obese children referred from primary care for suspected non-alcoholic fatty liver disease. Aliment Pharmacol Ther. (2013) 38:1267-77. doi: 10.1111/apt. 12518

32. Browning JD, Szczepaniak LS, Dobbins R, Nuremberg P, Horton JD, Cohen JC, et al. Prevalence of hepatic steatosis in an urban population in the United States: impact of ethnicity. Hepatology. (2004) 40:1387-95. doi: 10.1002/hep.20466

33. Vos MB, Abrams SH, Barlow SE, Caprio S, Daniels SR, Kohli R, et al. NASPGHAN clinical practice guideline for the diagnosis and treatment of nonalcoholic fatty liver disease in children: recommendations from the Expert Committee on NAFLD (ECON) and the North American Society of Pediatric Gastroenterology, Hepatology and Nutrition (NASPGHAN). J
Pediatr Gastroenterol Nutr. (2017) 64:319-34. doi: 10.1097/MPG.0000000000 001482

34. McMorrow AM, Connaughton RM, Lithander FE, Roche HM. Adipose tissue dysregulation and consequences in childhood and adolescent obesity: potential impact of dietary fat quality. Proc Nutr Soc. (2015) 74:67-82. doi: 10.1017/S002966511400158X

35. Cimini FA, Barchetta I, Carotti S, Bertoccini L, Baroni MG, VespasianiGentilucci U, et al. Relationship between adipose tissue dysfunction, vitamin $\mathrm{D}$ deficiency and the pathogenesis of non-alcoholic fatty liver disease. World J Gastroenterol. (2017) 23:3407-41. doi: 10.3748/wjg.v23.i19.3407

36. Marzuillo P, Del Giudice EM, Santoro N. Pediatric non-alcoholic fatty liver disease: new insights and future directions. World J Hepatol. (2014) 6:217-25. doi: 10.4254/wjh.v6.i4.217

37. Palmentieri B, de Sio I, La Mura V, Masarone M, Vecchione R, Bruno $\mathrm{S}$, et al. The role of bright liver echo pattern on ultrasound B-mode examination in the diagnosis of liver steatosis. Dig Liver Dis. (2006) 38:485-9. doi: 10.1016/j.dld.2006.03.021

Conflict of Interest: The authors declare that the research was conducted in the absence of any commercial or financial relationships that could be construed as a potential conflict of interest.

Copyright (C) 2019 Hua, Huang, Hu, Yao and Lai. This is an open-access article distributed under the terms of the Creative Commons Attribution License (CC BY). The use, distribution or reproduction in other forums is permitted, provided the original author(s) and the copyright owner(s) are credited and that the original publication in this journal is cited, in accordance with accepted academic practice. No use, distribution or reproduction is permitted which does not comply with these terms. 